\title{
Memory effects induced by initial switching conditions
}

\author{
J. Martorel* \\ Departament d'Estructura i Constituents de la Materia, Facultat Física, \\ University of Barcelona, Barcelona 08028, Spain \\ D. W. L. Sprung and W. van Dijk \\ Department of Physics and Astronomy, McMaster University, \\ Hamilton, Ontario L8S $4 M 1$ Canada \\ J. G. Muga $\dagger$ \\ Departamento de Química-Física, UPV-EHU, Apdo 644, 48080 Bilbao, Spain
}

\begin{abstract}
Initial-switching refers to the way in which the decay of an initially confined state begins, as the barrier isolating it from the exterior is relaxed. We study these effects in the context of Longhi's version of the Fano-Anderson model. Most authors assume the sudden approximation where the coupling is turned on instantaneously. We consider a finite rise time $T$, both numerically and analytically. When the coupling is ramped up linearly over a switching time $T$, we show that the asymptotic survival amplitude acquires a phase $T$ and is modulated by a factor $(\sin T) / T$. Several other results relating to the solution of the model are obtained. All site amplitudes have the same decay constant during the exponential decay regime. In the asymptotic regime, the amplitude and phase of decay oscillations depend on the initial-switching profile, but the period does not.
\end{abstract}

PACS numbers: 03.65.Wj, 21.10.Tg, 42.50.Xa

\section{INTRODUCTION}

Quantal effects in the time evolution of decaying systems have been studied since the beginning of quantum mechanics. Gamow's explanation of alpha-decay was among the first successful applications of quantum theory to radioactive nuclei [1]. The theory showed that decay was exponential in the accessible range of times, and related the lifetime to the nuclear charge $Z$ and decay energy. Khalfin [2] was the first to realize that, when the energy spectrum of the system is bounded from below, exponential decay cannot persist as $t \rightarrow \infty$. Eventually it has to be replaced by an asymptotic regime with a slower rate of decrease. Explicit solution of the Schrödinger equation for simple models of a particle escaping from a confining potential verified that in the asymptotic regime, the survival (or non-escape) probability shows power-law decrease [3]. Under rather general assumptions, this power-law can be shown to be $\propto t^{-3}$ for finite range potentials [4]. Some additional aspects are discussed in [5, 6], and references therein. Exponential decay is observed in a wide variety of physical systems. The predicted slower decay rate at long times has proven difficult to verify, but it is a universal feature present in theoretical models. This applies not only to a particle trapped by a potential barrier, but also to the decay of a discrete state coupled to a continuum [7, 8].

It is less well-known that when the confining barrier, or the coupling between discrete state and contin-

\footnotetext{
*Electronic address: martorell@ecm.ub.es
}

${ }^{\dagger}$ Electronic address: jg.muga@ehu.es uum, is time-dependent in the initial stages of decay, socalled "memory effects" affect the post-exponential survival probability of the system. These effects were proposed as a means of enhancing the survival probability, to make it feasible to observe the post-exponential regime experimentally. In particular, Robinson [9] considered adiabatic switching-on of the system over an infinite time period, to clarify the role of switching time versus observation time.

Tight binding models have been widely used in condensed matter for many years [10], 11] to describe tightly bound bands in crystals. More recently they have been applied to multilayered semiconductor heterostructures [12], to the dynamics of Bloch oscillations [13] and to the study of properties of Bose condensates in a lattice [14]. They are particularly well suited when the unit cell of the periodic struture leads to a well confined lowest energy state.

In this paper, we examine memory effects for finite switching times. We use a variant of the Fano-Anderson model [15, 16, 17] proposed by Longhi [18] to study deviations from exponential decay at short times, and the Zeno effect [19]. His model can be viewed as the decay of a discrete state coupled to a single-band tight-binding continuum. But, more interestingly from an experimental point of view, he presented it as a one dimensional semi-infinite periodic chain of discrete sites. The coupling between adjacent sites, $g(|n\rangle\langle n+1|+| n+1\rangle\langle n|)$, is described by a universal hopping constant, $g$, and the site energies are taken all to be equal. For two sites isolated from the rest, $\pi \hbar /(2 g)$ would be the half-period, the time for a complete transfer of occupation between the pair. Relative isolation of the first site is obtained by fixing its coupling parameter $g_{1} \equiv g \Delta$ to be different 
from the rest. Under these conditions, when at $t=0$ only site 1 is occupied, Longhi obtained the survival probability, its lifetime for exponential decay and the asymptotic power- law [18, 20]. The connection to models of a confined particle, like alpha-decay, views site 1 as the well, the ratio $\Delta=g_{1} / g$ as the effect of a confining barrier, and the semi- infinite chain of sites in position space as a single-band continuum. Fig. 11 illustrates the system

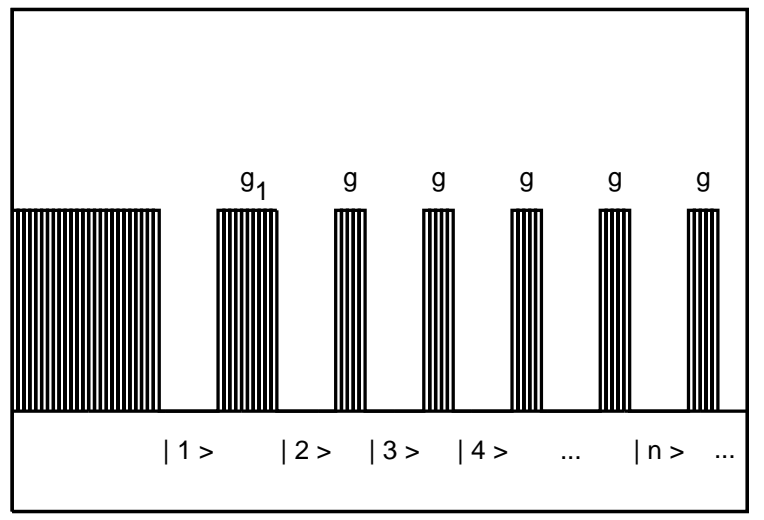

FIG. 1: Semi-infinite array of identical 1D wells coupled to a "defect" well separated by a thicker barrier.

we have in mind. The barrier separating site 1 from the rest of a semi-infinite array is thicker than the others, to reduce tunnelling, which in a tight binding model implies $g_{1}<g$. A thick enough barrier effectively makes $g_{1}=0$, removing site 1 from consideration.

An added interest of the model is that it has a classical electro-magnetic analogue, in the form of a waveguide array [21, 22]. Fig. 2 describes one such semi-infinte array with an initial "defect". Fig. 1(a) of [22] shows an experimental realization of such an array. Consider an ideal semi-infinite array of long parallel waveguides. The variation with $z$, (the longitudinal distance along the guide) of the electric field in the $n$ 'th guide, replaces the variation with time of the site amplitudes. Both systems obey a set of coupled mode equations formally the same as those for the time variation of the site amplitudes in the Fano-Anderson model. Recent experiments, using scanning tunnelling microscopy with sensitive nearfield probes, have allowed Longhi and collaborators [23] to measure the evanescent fields along the waveguides, and provide a convincing quantitative demonstration of the classical-quantal analogy for the case of a periodic parallel array. In addition the classical analogue of the quantum Zeno effect was verified [24]. The validity of the Fano-Anderson model to describe such effects has been quantitatively shown by these authors [23, 24].

In the present work, we study the problem of initial switching, by letting $g_{1}(t) / g \equiv \Delta_{v}(t)$ depend on time, during a finite initial time period $0<t<T$, following which $\Delta$ is constant. In Longhi's optical analogue, this

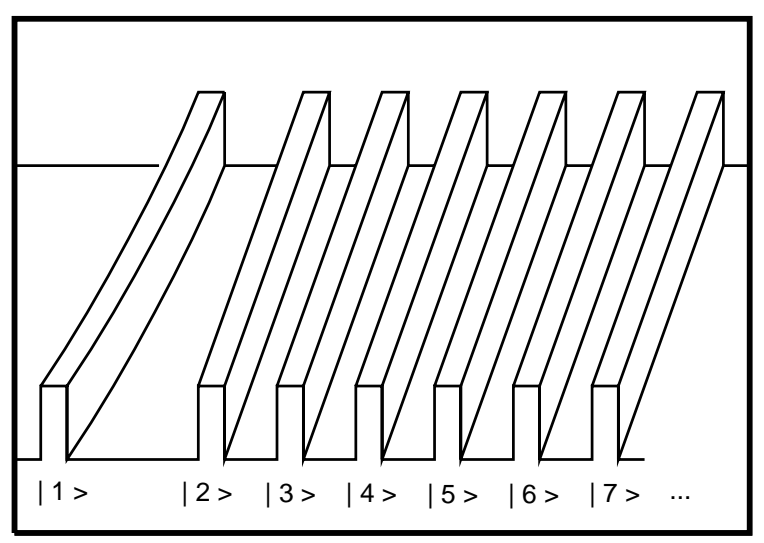

FIG. 2: A segment of a semi-infinite array of tunnel-coupled optical waveguides, made by etching. The $z$-axis is parallel to the guides, and $z_{T}$ at the TOP of the drawing. A logarithmic dependence on $z$ of the separation leads to linear dependence of the coupling.

could be implemented by having many parallel waveguides coupled to a first curved waveguide, with its separation distance decreasing smoothly with $z$ from an initially large value (where tunnelling is practically negligible), to a constant value beyond $z=z_{T}$. The final separation distance should be chosen to give well-expressed exponential decay. Longhi et al. [26] have demonstrated the experimental feasibility of a similar device (see their Fig. 1). Our work differs from that of Robinson [9], who considered a smooth switching function increasing adiabatically over $-\infty<t<\infty$, so his $\Delta_{R}(t)$ saturates only asymptotically. Finite switching times have also been considered in the context of threshold effects in pulsed laser photionization; see Chapter 6 of 25] for a review. In Section II we discuss solutions of Longhi's model in the exponential and asymptotic regimes. In Section III we derive analytic expressions for the time evolution during the period of varying coupling strength. Specific calculations of the effects are reported in Section IV. Verification of these predictions should be possible with present experimental capabilities. Verification of the predicted memory effects at long times may require further development of experimental techniques.

\section{DECAY FOR ARBITRARY INITIAL CONDITIONS}

To begin, we study the time evolution of a semi-infinite tight binding system that initially has a single site $n=q$ occupied at $t=0$. For convenience, we will use dimensionless units. Energies will be measured in units of $g$ (the hopping parameter of the periodic lattice), and time in units of $\hbar / g$. Wth the site energies set to zero, the 
Hamiltonian is

$$
\begin{aligned}
H & =-\Delta_{v}(t)[|1\rangle\langle 2|+| 2\rangle\langle 1|] \\
& -\sum_{n=2}^{\infty}[|n\rangle\langle n+1|+| n+1\rangle\langle n|] .
\end{aligned}
$$

We assume a positive coupling parameter $0<\Delta<1$. Longhi [18, 20] derived analytic expressions for the occupation amplitude $c_{n}(t)$, focussed primarily on the case that only the first site $q=1$ is occupied at time zero, and constant $\Delta$. Fig. 3 shows that the choice $\Delta=0.3$ gives a survival probability, $P(t)=\left|c_{1}(t)\right|^{2}$, which decays exponentially for a considerable range of times, and afterward the post-exponential decay consists of oscillations whose envelope follows the $t^{-3}$ law. This will be our example of reference in the remainder of the article.

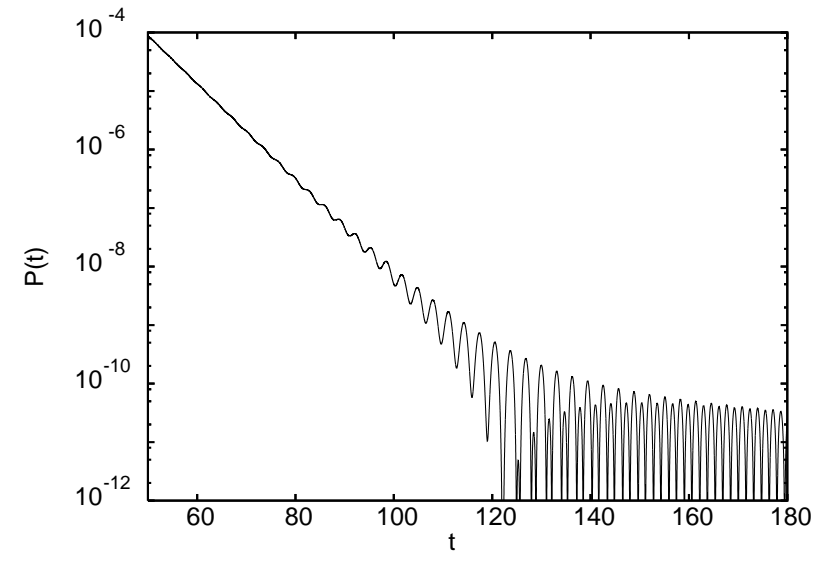

FIG. 3: Survival probability $P(t)$, for constant $\Delta=0.3$, in the region of changeover from exponential to asymptotic decay. Here and in the subsequent figures, time $t$ (dimensionless) is in units $\hbar / g$.

In this paper we suppose that during an initial period $0<t \leq T$, the coupling parameter $\Delta_{v}$ increases continuously from 0 to $\Delta$ and afterwards remains constant. In Section III we will study a specific choice for the initial time-dependence, that we call linear rise

$$
\begin{aligned}
\Delta_{v}(t)=\Delta_{\ell}(t) & \equiv t \Delta / T, & & t \leq T \\
& =\Delta, & & t>T .
\end{aligned}
$$

For this model we will derive explicit expressions for the survival amplitude and other quantities, valid to lowest order in $\Delta$. Exponential decay over a sizeable time scale requires that $\Delta$ be small compared to 1 , so linear terms should suffice to establish the importance of such effects.

Initially we focus on general properties that apply to any scheme for the initial switching-on of $\Delta$. Given any profile $\Delta_{v}(t)$, the site occupation amplitudes, $c_{n}(t), n=$ $1,2, \cdots$ can be determined by numerical solution of the time-dependent Schrödinger equation (TDSE), for which we use the method described in [27]. Alternatively, in Sec. III we use the time evolution operator to derive approximate analytic results. In either approach, the end result is a set of occupation amplitudes at $t=T$ which in this Section we assume to be known, and that we write in vector form

$$
|\vec{\vartheta}\rangle=\left(\begin{array}{llllll}
\vartheta_{1} & \vartheta_{2} & \ldots & \cdots & \vartheta_{q} & \cdots
\end{array}\right)^{\top}
$$

with $\sum_{q=1}^{\infty}\left|\vartheta_{q}\right|^{2}=1$ and where by $\mathrm{T}$ we denote the transpose. Since we will always assume that after time $T$, $\Delta_{v}(t \geq T)=\Delta$ remains constant, it is convenient to reset the zero of time by writing $\tilde{t}=t-T$. In the remainder of this section we study the time evolution of a system with these evolved initial conditions, at $\tilde{t}=0$, given by Eq. (3), and constant $\Delta$. The further evolution is governed by the TDSE

$$
i \frac{\partial}{\partial \tilde{t}}|\vec{\psi}(\tilde{t})\rangle=H|\vec{\psi}(\tilde{t})\rangle
$$

with

$$
|\vec{\psi}(\tilde{t})\rangle=\left(c_{1}(\tilde{t}), c_{2}(\tilde{t}), . ., c_{n}(\tilde{t}), . .\right)^{\top} .
$$

The linearity in time of the TDSE implies that

$$
c_{n}(\tilde{t})=\sum_{q=1}^{\infty} \vartheta_{q} c_{n, q}(\tilde{t})
$$

where the $c_{n, q}(\tilde{t})$ are the site amplitudes of a state, $\left|\vec{\psi}_{q}(\tilde{t})\right\rangle$, which at $\tilde{t}=0$ has unit real amplitude $c_{q, q}(0)=1$ on the $q$-th site, and vanishes on all others.

$$
\begin{aligned}
\left|\vec{\psi}_{q}(\tilde{t}=0)\right\rangle^{\top} & =(0,0, \cdots, 1,0, \cdots) \\
\left|\vec{\psi}_{q}(\tilde{t})\right\rangle^{\top} & =\left(c_{1, q}(\tilde{t}), c_{2, q}(\tilde{t}), \cdots c_{n, q}(\tilde{t}), \cdots\right)
\end{aligned}
$$

Stationary solutions. To determine these $c_{n, q}(\tilde{t})$, we first find stationary solutions of the coupled equations, and then propagate linear combinations of them in time. Writing $|\vec{\psi}(\tilde{t})\rangle=\exp (-i E \tilde{t})|\vec{u}\rangle$, the components of $|\vec{u}\rangle$ satisfy

$$
\begin{aligned}
& 0=2 \cos \phi u_{1}-\Delta u_{2} \\
& 0=-\Delta u_{1}+2 \cos \phi u_{2}-u_{3} \\
& 0=-u_{n-1}+2 \cos \phi u_{n}-u_{n+1} \quad, \quad n \geq 3
\end{aligned}
$$

where we have written $E \equiv-2 \cos \phi$. The solutio is most easily obtained by expressing $u_{n}$ in terms of incoming and outgoing waves with amplitudes denoted $\mathcal{I}$ and $\mathcal{O}$ :

$$
\begin{aligned}
u_{n} & =\mathcal{I} e^{i(n-2) \phi}-\mathcal{O} e^{-i(n-2) \phi} \quad, n \geq 2 \\
u_{1} & =\Delta(\mathcal{I}-\mathcal{O}) /(2 \cos \phi) \\
\Delta u_{1} & =2 \cos \phi u_{2}-u_{3} .
\end{aligned}
$$

Inserting the first and second lines into the third, gives

$$
\frac{\mathcal{O}}{\mathcal{I}}=\frac{2 \cos \phi e^{-i \phi}-\Delta^{2}}{2 \cos \phi e^{i \phi}-\Delta^{2}}=\frac{\alpha^{2}+e^{-2 i \phi}}{\alpha^{2}+e^{+2 i \phi}} \equiv r(\phi),
$$

where $\alpha^{2} \equiv 1-\Delta^{2}$ is the decoupling parameter. Since the normalization of the states $|\vec{u}\rangle$ is arbitrary at this stage, 
we may define the amplitudes $\mathcal{O}$ and $\mathcal{I}$ as the numerator and denominator of Eq. (10). Then the $u_{n}$ in Eq. (9) take the compact form

$$
\begin{aligned}
& u_{n}(\phi)=2 i\left(\sin n \phi+\alpha^{2} \sin (n-2) \phi\right), \quad n \geq 2 \\
& u_{1}(\phi)=2 i \Delta \sin \phi
\end{aligned}
$$

(Notice that if line one is specialized to $n=1$, we obtain $\Delta$ times $u_{1}(\phi)$. Therefore one can avoid treating $n=1$ as a special case, if we simply remember to remove this extra factor.)

Time dependent solutions: Following 20] we write the $c_{n}(\tilde{t})$ as weighted integrals over the stationary solutions, with a weight function $F(\phi)$ (still to be determined) expressed as a Fourier series

$$
\begin{aligned}
F(\phi) & \equiv \frac{1}{2 \pi \Delta} \sum_{N=1}^{\infty} T_{N} e^{-i N \phi} \\
c_{n}(\tilde{t}) & =\int_{-\pi}^{\pi} d \phi u_{n}(\phi) e^{-i E \tilde{t}} F(\phi) .
\end{aligned}
$$

The parameters $T_{N}$ are determined by the initial conditions, as we now explain. In what follows we will often use properties of Bessel functions which can be found in Chapter 11 of Arfken [28]; to avoid tiresome repetition, we will cite it no further. For convenience we introduce symbols $\mathcal{J}_{q}(2 t) \equiv i^{q} J_{q}(2 t)$, which have the symmetry $\mathcal{J}_{q}=\mathcal{J}_{-q}$, and satisfy the recurrence relation

$$
\mathcal{J}_{n-1}-\mathcal{J}_{n+1}=\frac{n}{i t} \mathcal{J}_{n}
$$

This allows us to write

$$
\begin{aligned}
I_{p, N}= & \frac{i}{\pi} \int_{-\pi}^{\pi} d \phi \sin p \phi e^{i(2 \tilde{t} \cos \phi-N \phi)} \\
= & i^{N-p} J_{N-p}(2 \tilde{t})-i^{N+p} J_{N+p}(2 \tilde{t}) \\
\equiv & \mathcal{J}_{N-p}(2 \tilde{t})-\mathcal{J}_{N+p}(2 \tilde{t}) \\
= & \mathcal{J}_{N-p}-\mathcal{J}_{N-p+2}+\mathcal{J}_{N-p+2}-\mathcal{J}_{N-p+4}+ \\
& \quad \cdots+\mathcal{J}_{N+p-2}-\mathcal{J}_{N+p} \\
= & \sum_{m=1}^{p} \frac{(N+p+1-2 m)}{i \tilde{t}} \mathcal{J}_{N+p+1-2 m}
\end{aligned}
$$

In the limit of large argument $2 t \gg N+p, I_{p, N}$ approaches its asymptotic form

$$
\begin{aligned}
I_{p, N}^{(a)} & \equiv \sum_{m=1}^{p} \frac{(N+p+1-2 m)}{\sqrt{\pi} \tilde{t}^{3 / 2}} i^{N+p-2 m} \\
& =\sum_{m=1}^{p} \frac{(N+p+1-2 m)}{\sqrt{\pi} \tilde{t}^{3 / 2}} i^{N+p} \times \\
& =\frac{i^{N+p-2}}{\sqrt{\pi} \tilde{t}^{3 / 2}} \sum_{m=1}^{p}(N+p+1-2 m) \times \\
& =\frac{i^{N+p-2}}{\sqrt{\pi} \tilde{t}^{3 / 2}} N p \cos [2 \tilde{t}-(N+p-1 / 2) \pi / 2] \\
&
\end{aligned}
$$

Using Eqs. (11), (12) and (14),

$$
\begin{aligned}
c_{n}(\tilde{t})= & \frac{1}{\Delta} \sum_{N=1} T_{N}\left[\left(\mathcal{J}_{N-n}(2 \tilde{t})-\mathcal{J}_{N+n}(2 \tilde{t})\right)\right. \\
& \left.+\alpha^{2}\left(\mathcal{J}_{N-n+2}(2 \tilde{t})-\mathcal{J}_{N+n-2}(2 \tilde{t})\right)\right] \\
c_{1}(\tilde{t})= & \sum_{N=1} T_{N}\left(\mathcal{J}_{N-1}(2 \tilde{t})-\mathcal{J}_{N+1}(2 \tilde{t})\right) \\
= & \sum_{N=1} T_{N} \frac{N}{i \tilde{t}} \mathcal{J}_{N}(2 \tilde{t}) \\
= & \sum_{N=1} T_{N} i^{N-1} \frac{N}{\tilde{t}} J_{N}(2 \tilde{t})
\end{aligned}
$$

so that at $\tilde{t}=0$ :

$$
\begin{aligned}
\Delta c_{n}(0) & =\left(T_{n}+\alpha^{2} T_{n-2}\right) \quad, \quad n \geq 2 \\
c_{1}(0) & =T_{1} .
\end{aligned}
$$

Note that $T_{0}=0$, since only the sites with positive indices exist. Given initial conditions for the $c_{n}(\tilde{t})$, as e.g. in Eq. (3), one determines the $T_{N}, N=1,2, .$. from the recurrence relation Eq. (18). This completes construction of the time-dependent solution.

Up to now, our results are valid for all times $\tilde{t}>0$. In the next two subsections we develop approximations valid in the asymptotic and exponential regimes which are illustrated in Fig. 1.

\section{A. Asymptotic approximation}

Since $J_{N}(2 \tilde{t}) / \tilde{t} \propto(\tilde{t})^{-3 / 2}$ when $\tilde{t} \rightarrow \infty$, one sees that for long times $\left|c_{1}(\tilde{t})\right|^{2} \propto \tilde{t}^{-3}$ for any set of initial occupations, as stated in the Introduction. Using Eqs. (16) and 
(17) we find

$$
\begin{aligned}
& c_{1}(\tilde{t}) \simeq c_{1}^{(a)}(\tilde{t}) \\
= & \frac{1}{\sqrt{\pi} \tilde{t}^{3 / 2}} \sum_{N=1} N T_{N} i^{N-1} \cos \left(2 \tilde{t}-N \frac{\pi}{2}-\frac{\pi}{4}\right) \\
= & \frac{1}{\sqrt{\pi} \tilde{t}^{3 / 2}}\left[\left(\sum_{l=0}(2 l+1) T_{2 l+1}\right) \sin \left(2 \tilde{t}-\frac{\pi}{4}\right)\right. \\
& \left.-i\left(\sum_{l=0} 2 l T_{2 l}\right) \cos \left(2 \tilde{t}-\frac{\pi}{4}\right)\right],
\end{aligned}
$$

where for later convenience we have isolated the contributions from even and odd order $T_{N}$. Providing that the $T_{N}$ are real, (which they will be,) this is equivalent to separating the real and imaginary parts.

For the asymptotic behaviour of the $c_{n}, n \geq 2$, we use Eqs. (14), (16) and (17) to arrive at

$$
\begin{aligned}
& c_{n}^{(a)}(\tilde{t})=\frac{i^{n-1}}{\sqrt{\pi} \tilde{t}^{3 / 2} \Delta}\left(n+\alpha^{2}(n-2)\right) \times \\
& \sum_{N=1} i^{N-1} N T_{N} \cos \left(2 \tilde{t}-\left(N+n-\frac{1}{2}\right) \frac{\pi}{2}\right) \\
& =\frac{i^{n-1}\left(n+\alpha^{2}(n-2)\right)}{\sqrt{\pi} \tilde{t}^{3 / 2} \Delta} \times \\
& {\left[\left(\sum_{l=0}(2 l+1) T_{2 l+1}\right) \cos \left(2 \tilde{t}-(n+1 / 2) \frac{\pi}{2}\right)\right.} \\
& \left.-i\left(\sum_{l=0} 2 l T_{2 l}\right) \cos \left(2 \tilde{t}-(n-1 / 2) \frac{\pi}{2}\right)\right],
\end{aligned}
$$

for $n \geq 2$. Providing that $2 \tilde{t}>>n \pi$, we can replace the cosines in the last lines by $J_{n}(2 \tilde{t})$ and $J_{n-1}(2 \tilde{t})$ respectively, (making appropriate adjustments to the prefactor).

Now we particularise to cases where the initial amplitude is confined to a single site labelled $q$. By combining these results, we can finally write the general solution for arbitrary initial occupations.

Case 1: Initial amplitude only on site 1. At $\tilde{t}=0$ : $c_{1}(0)=1, c_{n}(0)=0, n \geq 2$. It is then immediate from Eq. (18) that $T_{2 l}=0$ for all $l$ and that $T_{2 l+1}=(-)^{l} \alpha^{2 l}$. Inserting these in Eq. (19) one finds

$$
\begin{aligned}
c_{1,1}^{(a)}(\tilde{t}) & =\frac{1}{\sqrt{\pi} \tilde{t}^{3 / 2}} \sin \left(2 \tilde{t}-\frac{\pi}{4}\right) \sum_{l=0}(2 l+1)(-)^{l} \alpha^{2 l} \\
& =\frac{1}{\sqrt{\pi} \tilde{t}^{3 / 2}} \frac{1-\alpha^{2}}{\left(1+\alpha^{2}\right)^{2}} \sin \left(2 \tilde{t}-\frac{\pi}{4}\right)
\end{aligned}
$$

in agreement with Eq. 8 of [18]. Hereafter we will call this case the "sudden approximation" because it corresponds to the abrupt switching-on of the coupling from $\Delta_{v}=0$ to $\Delta_{v}=\Delta$ at $t=T=0$.
Case 2: Initial amplitude on another odd site. Consider now $c_{n}(0)=\delta_{n, q}, q>1$ odd. Then $T_{2 l}=0, l=$ $0,1, .$. and

$$
\begin{aligned}
T_{1} & =T_{3}=\cdots=T_{q-2}=0 \quad, \quad T_{q}=\Delta \\
T_{q+2 l} & =(-)^{l} \alpha^{2 l} \Delta,
\end{aligned}
$$

so that Eq. (19) gives

$$
c_{1, q}^{(a)}(\tilde{t})=\frac{\Delta}{\sqrt{\pi} \tilde{t}^{3 / 2}} \frac{q\left(1+\alpha^{2}\right)-2 \alpha^{2}}{\left(1+\alpha^{2}\right)^{2}} \sin \left(2 \tilde{t}-\frac{\pi}{4}\right)
$$

Note that for $q=1$ this result agrees with Eq. (21), if we remove the explicit $\Delta$, in agreement with our earlier remark. The meaning of the factor $\Delta$ is this: When site 1 is initially empty, the wave must cross the barrier at least once before its decay may begin.

Case 3: Initial amplitude on an even site. When $c_{n}(0)=\delta_{n, q}, q$ even, we find $T_{2 l+1}=0, l=0,1, \cdots$ and

$$
\begin{aligned}
T_{0} & =T_{2}=\cdots=T_{q-2}=0 \quad, \quad T_{q}=\Delta \\
T_{q+2 l} & =(-)^{l} \alpha^{2 l} \Delta .
\end{aligned}
$$

From Eq. (19)

$$
c_{1, q}^{(a)}(\tilde{t})=\frac{-i \Delta}{\sqrt{\pi} \tilde{t}^{3 / 2}} \frac{q\left(1+\alpha^{2}\right)-2 \alpha^{2}}{\left(1+\alpha^{2}\right)^{2}} \cos \left(2 \tilde{t}-\frac{\pi}{4}\right)
$$

General initial conditions. We now combine the previous results to obtain the most general solution. Inserting Eqs. (23) and (25) into (6) we find

$$
\begin{aligned}
& c_{1}^{(a)}(\tilde{t})=\frac{1}{\sqrt{\pi}\left(1+\alpha^{2}\right)^{2} \tilde{t}^{3 / 2}} \\
& \quad \times\left[S_{o} \sin \left(2 \tilde{t}-\frac{\pi}{4}\right)-i S_{e} \cos \left(2 \tilde{t}-\frac{\pi}{4}\right)\right]
\end{aligned}
$$

with the amplitudes summed over odd (even) indices

$$
S_{o(e)} \equiv \sum_{q=o d d(\text { even })}^{\infty} \tilde{\vartheta}_{q}\left[q\left(1+\alpha^{2}\right)-2 \alpha^{2}\right]
$$

where we have defined $\tilde{\vartheta}_{1}=\vartheta_{1}$ and $\tilde{\vartheta}_{q}=\vartheta_{q} \Delta, q=$ $2,3, \cdots$.

In principle, the phases of the amplitudes $\vartheta_{q}$ at time zero are arbitrary, so that $S_{0}$ and $S_{e}$ are neither purely real or imaginary. Then $c_{1}^{(a)}$ of Eq. (26) is a complex number and will have a node only accidentally. But in the case we wish to discuss, it is assumed that at some time in the past the system was prepared with only site 1 occupied and $c_{1}(t=0)=1$. During the switching on process, the system evolved to build up the amplitudes in sites $2,3, \cdots$. In the special case of sudden switching, $T=0$, we can apply Eq. (20) with $\tilde{t}=t$ to see that the resulting phase of $c_{q}(t)$ is $i^{q-1}$, the quantities $T_{N}$ being real. Using a perturbation expansion, we will see that the same phases are generated by the time-evolution operator 
in Section III (see discussion following Eq. (61)). As a result, the amplitudes on odd-numbered sites are real and those on even numbered sites, pure imaginary. Hence $S_{o}$ and $X_{e}=-i S_{e}$ are both real, allowing us to write

$$
\begin{aligned}
c_{1}^{(a)}(\tilde{t}) & =\frac{1}{\sqrt{\pi}\left(1+\alpha^{2}\right)^{2} \tilde{t}^{3 / 2}} \mathcal{A} \sin \left(2 \tilde{t}-\frac{\pi}{4}+\Phi\right) \\
\mathcal{A} & =\sqrt{S_{o}^{2}+X_{e}^{2}} \\
\Phi & =\arctan \left(X_{e} / S_{o}\right) .
\end{aligned}
$$

This simplification ensures that the survival probability will still have nodes, and the period of the asymptotic oscillations is not changed by the initial switching process. Compared to the sudden approximation Eq. (21), there is an extra phase $\Phi$, and the asymptotic amplitude involves $\mathcal{A}$ in place of $\Delta$. To have $\Phi=0$ would require that the even-sites are empty at $\tilde{t}=0$.

\section{B. Exponential decay regime}

Again, we first consider the situation where at $\tilde{t}=0$, a single site $q$ is occupied, and use these as building blocks for the general result to be given at the end. Inserting the expressions Eqs. (22) or (24) into Eq. (12)

$$
F_{q}(\phi)=\frac{1}{2 \pi z^{q}} \frac{z^{2}}{z^{2}+\alpha^{2}},
$$

where we have set $z=\exp (i \phi)$ and the subindex $q$ refers to the initial condition, $c_{n}(0)=\delta_{q, n}$. We note that $F_{q}(\phi)$ has poles at the origin and $z_{p}= \pm i \alpha$. In terms of $z$, Eq. (12) becomes:

$$
c_{k}(\tilde{t})=\frac{1}{2 \pi i} \oint d z e^{i \tilde{t}(z+1 / z)} \frac{u_{k}(z)}{z^{q-1}} \frac{1}{z^{2}+\alpha^{2}},
$$

the path of integration being the unit circle. As shown by Longhi [18], Eqs. (9) to (12), to extract the exponentially-decaying contribution to $c_{k}(t)$ one may use the residue theorem to evaluate the integral, retaining only the contribution from the Gamow pole at $z_{g}=-i \alpha$. The conjugate pole corresponds to exponential growth, which is not the physical process, and the poles at the origin give compensating contributions, leaving only a small remainder, at least in the survival probability. The Gamow pole contributes

$$
c_{k, q}^{(e)}(\tilde{t})=\frac{u_{k}\left(z_{g}\right)}{2(-i \alpha)^{q}} e^{-\gamma \tilde{t} / 2}
$$

where the superscript (e) means exponential decay, and

$$
\frac{\gamma}{2} \equiv \frac{1-\alpha^{2}}{\alpha}=\frac{\Delta^{2}}{\alpha}
$$

This is a remarkable result: it shows that all the initial conditions of the form $c_{n}(0)=\delta_{n, q}$ lead to the same lifetime, $\tau=1 / \gamma$, for the exponentially decaying contribution to $\left|c_{k, q}(\tilde{t})\right|^{2}$, no matter which site $q$ is initially occupied.

We develop the above result by evaluating $u_{k}\left(z_{g}\right)$. From Eq. (11), for $k \geq 2$,

$$
u_{k}\left(z_{g}\right)=-i^{k} \frac{1-\alpha^{4}}{\alpha^{k}},
$$

and therefore:

$$
c_{k, q}^{(e)}(\tilde{t})=-i^{k+q} \frac{\Delta^{2}\left(1+\alpha^{2}\right)}{2 \alpha^{k+q}} e^{-\gamma \tilde{t} / 2}, \quad k \geq 2 .
$$

When $k=1$, the same calculation has a factor $1 / \Delta$ in comparison:

$$
u_{1}\left(z_{g}\right)=-i \Delta \frac{1+\alpha^{2}}{\alpha}
$$

and

$$
c_{1, q}^{(e)}(\tilde{t})=-i^{q+1} \frac{\Delta\left(1+\alpha^{2}\right)}{2 \alpha^{q+1}} e^{-\gamma \tilde{t} / 2} .
$$

The sudden approximation, Case 1 , with $c_{1}(0)=1$, requires a separate discussion since $T_{2 l}=0$ and $T_{2 l+1}=$ $(-)^{2} \alpha^{2 l}$, without the factor $\Delta$ of Eq. (22). One now finds

$$
F_{1}(\phi)=\frac{z}{2 \pi \Delta\left(z^{2}+\alpha^{2}\right)}
$$

and

$$
c_{k, 1}^{(e)}(\tilde{t})=i^{k-1} \frac{\Delta\left(1+\alpha^{2}\right)}{2 \alpha^{k+1}} e^{-\gamma \tilde{t} / 2}
$$

when $k \geq 2$, while

$$
c_{1,1}^{(e)}(\tilde{t})=\frac{1+\alpha^{2}}{2 \alpha^{2}} e^{-\gamma \tilde{t} / 2} .
$$

This last result agrees with Eq. (11) of [18].

Finally, we write the amplitudes for general initial occupations. Inserting the above results in Eq. (6) gives

$$
\begin{aligned}
& c_{1}^{(e)}(\tilde{t})=\frac{1}{2 i \alpha}\left(1+\alpha^{2}\right)\left(\sum_{q=1}^{\infty} \tilde{\vartheta}_{q} \frac{i^{q}}{\alpha^{q}}\right) e^{-\gamma \tilde{t} / 2}, \\
& c_{k}^{(e)}(\tilde{t})=\frac{i^{k-2}}{2 \alpha^{k}} \Delta\left(1+\alpha^{2}\right)\left(\sum_{q=1}^{\infty} \tilde{\vartheta}_{q} \frac{i^{q}}{\alpha^{q}}\right) e^{-\gamma \tilde{t} / 2},
\end{aligned}
$$

when $k \geq 2$. Clearly the exponential decay contribution has a delay $T$, manifest in the appearance of $\tilde{t}=t-T$ in these expressions. Furthermore, one sees that the decay constant $\gamma$ depends neither on the initial conditions nor on the site considered.

\section{INITIAL SWITCHING}

We now address our main objective, which is time evolution when the coupling between site 1 and site $2, \Delta_{v}(t)$, 
varies continuously with time. To do so we introduce a simple diagrammatic technique which describes visually the action of the time evolution operator. To introduce these diagrams we first apply them to the oft-studied case of "sudden switching": $\Delta_{v}(t>0)=\Delta=$ constant, which will serve as a reference for results with variable $\Delta$.

The time evolution operator can be written as

$$
\begin{aligned}
U(t) & =\sum_{m=0}^{\infty} U_{m}(t) \quad \text { with } \\
U_{m}(t) & =(-i)^{m} \int_{0}^{t} d t_{m} \int_{0}^{t_{m}} d t_{m-1} \cdots \int_{0}^{t_{2}} d t_{1} \\
& \times H\left(t_{m}\right) H\left(t_{m-1}\right) \cdots H\left(t_{1}\right)
\end{aligned}
$$

and in the special case of a time independent $H$, this reduces to $U(t)=\exp (-i H t)=\sum_{m=0}^{\infty}(-i t H)^{m} /(m !)$. Suppose we start with the state $\mid 1>$ with only site 1 occupied initially. Then

$$
U(t)|1\rangle=|\Psi(t)\rangle=\left(\begin{array}{c}
c_{1}(t) \\
c_{2}(t) \\
\vdots
\end{array}\right)
$$

\section{A. Diagrammatic method and sudden switching}

We now develop a diagrammatic method to calculate the time evolution operator, first in the context of sudden switching where the exact solution is already available. It will be extended to linear switching in the next subsection. To begin we compute $U_{m}(t)|1\rangle$ for increasing values of $m$, assuming $\Delta$ is constant. For $m=0, U_{0}(t)=1$, and therefore

$$
U_{0}(t)|1\rangle=|1\rangle
$$

When $m=1$

$$
\begin{aligned}
U_{1}(t)|1\rangle & =-i \int_{0}^{t} d t_{1} H|1\rangle=-i t H|1\rangle \\
& =i t \Delta|2\rangle .
\end{aligned}
$$

The first graph in Fig. 4 describes this process: the circles represent the sites, the time evolution is downwards and the lines describe hopping between adjacent sites. The $m=2$ contribution is

$$
\begin{aligned}
& U_{2}(t)=(-i)^{2} \int_{0}^{t} d t_{2} \int_{0}^{t_{2}} d t_{1} H^{2}=\frac{(-i)^{2}}{2 !} t^{2} H^{2} \\
& U_{2}(t)|1\rangle=\frac{(-i t)^{2}}{2 !}\left(\Delta^{2}|1\rangle+\Delta|3\rangle\right)
\end{aligned}
$$

and the two corresponding diagrams are in the lower row of Fig. 4. Continuation to higher $m$ is straightforward. The rules to generate the contributions to $U_{m}(t)|1\rangle$ are: 1) each line joining 1 and 2 contributes a factor $-\Delta$, whereas the other lines contribute a factor -1 each; 2 ) if the diagram has $m$ lines: multiply by $(-i t)^{m} /(m !)$.
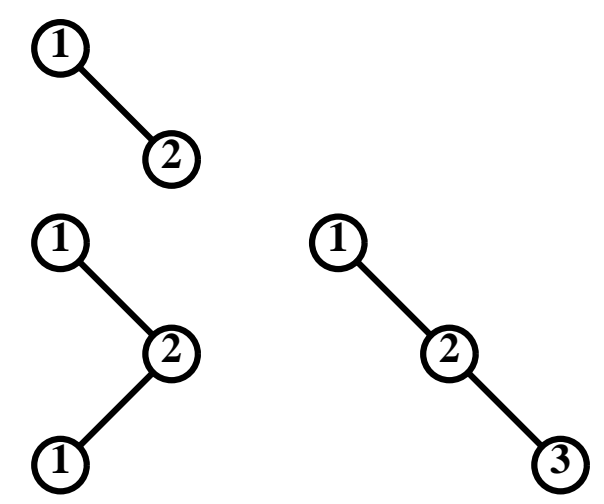

FIG. 4: Diagrams which begin at site 1 , with $m=1$ and 2 lines. The meaning of the various symbols is explained in text.

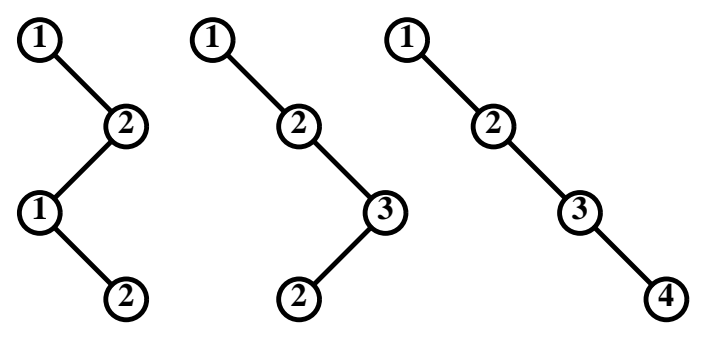

FIG. 5: Three line diagrams which begin at site 1 .

Combining these two, the net result is to multiply by $(i t)^{m} /(m !)$. The final state is the last vertex at bottom of the diagram. As a further example consider the $m=3$ contributions: applying the rules to the three graphs of Fig. 5] one can immediately write

$$
U_{3}(t)|1\rangle=\frac{(i t)^{3}}{3 !}\left(\Delta^{3}|2\rangle+\Delta|2\rangle+\Delta|4\rangle\right) .
$$

We now focus on contributions to $c_{1}(t)$, to all orders in $m$. The relevant diagrams will be those having $|1\rangle$ as both initial and final state. Since this implies an even number of lines, we write $m=2 l$ with $l=1,2, \cdots$. The simplest of these diagrams, $l=1$, is already included in Fig. 4. The two diagrams with $l=2$ are shown in Fig. 6. In the special case $\Delta=1$, each diagram with the same number of lines gives an identical contribution to $c_{1}$. Applying the general rules, the desired series is

$$
c_{1}(t, \Delta=1)=\sum_{l=0}^{\infty}(-)^{l} \frac{t^{2 l}}{(2 l) !} N_{2 l}^{1 \rightarrow 1},
$$



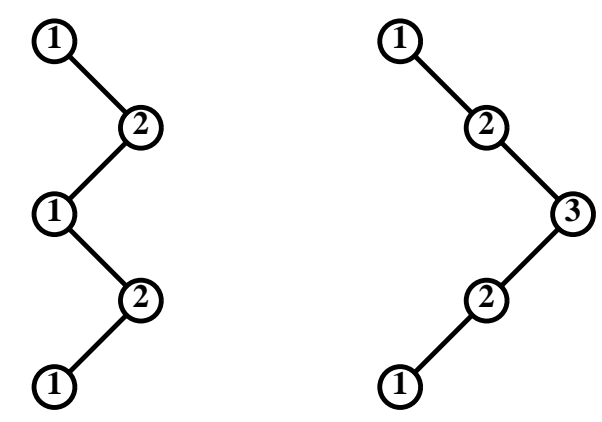

FIG. 6: Four line diagrams which begin and end at site 1 .

where $N_{2 l}^{1 \rightarrow 1}$ is the number of distinct diagrams with $2 l$ lines having $|1\rangle$ as both initial and final state. To determine it, we rewrite Eq. (17) for the Case 1 initial conditions and $\Delta=1$ : it simplifies to

$$
c_{1}(t, \Delta=1)=\frac{J_{1}(2 t)}{t}=\sum_{l=0}^{\infty} \frac{(-)^{l} t^{2 l}}{l !(l+1) !},
$$

using the Taylor series for $J_{1}(2 t)$. Comparing Eqs. (47) and (48), it follows that

$$
N_{2 l}^{1 \rightarrow 1}=\frac{(2 l) !}{l !(l+1) !} .
$$

Now that the $N_{l}^{1 \rightarrow 1}$ are known, we can return to the more general case $\Delta \neq 1$ and consider those diagrams that give contributions of order $\Delta^{2}$ to $c_{1}(t)$. These begin and end at $|1\rangle$, but never have $|1\rangle$ as an intermediate state. The second diagram in Fig. 6 is an example. It is now easy to write down their contribution:

$$
c_{1}^{(2)}=\Delta^{2} \sum_{l=1}^{\infty} \frac{(i t)^{2 l}}{(2 l) !} N_{2(l-1)}^{1 \rightarrow 1}=\Delta^{2} \sum_{l=1}^{\infty} \frac{(-)^{l} t^{2 l}}{(4 l-2)(l !)^{2}}
$$

Here we have noted that the number of diagrams with $2(l-1)$ lines and beginning and ending at $|2\rangle$ without |1) as intermediate states, equals $N_{2(l-1)}^{1 \rightarrow 1}$. Since we are interested in values of $\Delta \ll 1$, we will not write down expressions for contributions of order $\Delta^{4}$ or higher. This supposes that processes with more than two tunneling events between sites 1 and 2 can be neglected during the short initial-switching time interval.

To find the amplitude for site 2 we begin by considering again the special case $\Delta=1$. The simplest diagrams that connect $|1\rangle$ to $|2\rangle$ are shown in Fig. 4 (in the upper row) and Fig. 5 (the first two). They have an odd number of lines, $m=2 l+1, l=0,1,2, \cdots$. Again all the diagrams with the same number of lines give equal contributions. Thus

$$
c_{2}(t, \Delta=1)=\sum_{l=0} \frac{(i t)^{2 l+1}}{(2 l+1) !} N_{2 l+1}^{1 \rightarrow 2} .
$$

Eq. (17) tells us that when $\Delta=1\left(\alpha^{2}=0\right)$ and Case 1 initial conditions,

$$
c_{2}(t, \Delta=1)=\frac{2 i}{t} J_{2}(2 t)=2 i t \sum_{l=0} \frac{\left(-t^{2}\right)^{l}}{l !(l+2) !}
$$

and therefore

$$
N_{2 l+1}^{1 \rightarrow 2}=\frac{2(2 l+1) !}{l !(l+2) !} .
$$

We now return to the case $\Delta \neq 1$, and determine the contribution to $c_{2}$ linear in $\Delta$. The simplest diagrams are those in the top row of Fig. 4, and in Fig. 5. More generally: we include all diagrams that begin with a link from 1 to 2 and are completed with lines that go from 2 to 2 without having 1 as an intermediate state. All such diagrams have an odd number $n=2 l+1$ of lines, $l=0,1, \cdots$ and their contributions are

$$
\begin{aligned}
c_{2}^{(1)}(t) & =\Delta \sum_{l=0}^{\infty} \frac{(i t)^{2 l+1}}{(2 l+1) !} N_{2 l}^{1 \rightarrow 1} \\
& =i \Delta \sum_{l=0}(-)^{l} \frac{t^{2 l+1}}{(2 l+1) l !(l+1) !}
\end{aligned}
$$

where we have used that the number of the above subdiagrams, beginning and ending at 2, equals $N_{2 l}^{1 \rightarrow 1}$.

Finally, to determine the amplitudes for site $n$ we proceed similarly: For constant $\Delta=1$,

$$
c_{n}(t, \Delta=1)=\sum_{l=0}^{\infty}(-)^{l} \frac{(i t)^{n-1+2 l}}{(n-1+2 l) !} N_{n-1+2 l}^{1 \rightarrow n}
$$

and comparison to Eq. (17) with Case 1 initial conditions

$$
\begin{aligned}
c_{n}(t, \Delta=1) & =i^{n-1} \frac{n}{t} J_{n}(2 t) \\
& =i^{n-1} n \sum_{l=0}(-)^{l} \frac{t^{n-1+2 l}}{l !(n+l) !}
\end{aligned}
$$

shows that

$$
N_{n-1+2 l}^{1 \rightarrow n}=\frac{n(n-1+2 l) !}{l !(n+l) !}
$$

which generalizes Eqs. (49) and (53). Finally, for constant $\Delta \neq 1$ we obtain the contribution to $c_{n}$ linear in $\Delta$

$$
c_{n}^{(1)}(t)=\Delta \sum_{l=0}^{\infty} \frac{n-1}{n-1+2 l} \frac{(i t)^{n-1+2 l}}{l !(n-1+l) !} .
$$

\section{B. Linear switching}

When $\Delta(t)$ depends on time, the time evolution operator is given by Eq. (41) and the term of order $m$ involves $m$ consecutive integrations over intermediate times. In 
computing the contribution linear in $\Delta$ to $c_{n}(t)$, the timedependent term will appear only in the first line of the diagram. Thus for $m=1$

$$
U_{1}\left(t_{2}\right)|1\rangle=i \int_{0}^{t_{2}} d t_{1} \Delta_{v}\left(t_{1}\right)|2\rangle \equiv f\left(t_{2}\right)|2\rangle
$$

and we construct the contributions from the diagrams corresponding to higher orders by integrating $f(t)$ the required number of times.

$$
\mathcal{I}_{m}(t)=\int_{0}^{t} d t_{m} \int_{0}^{t_{m-1}} d t_{m-1} \cdots \int_{0}^{t_{3}} d t_{2} f\left(t_{2}\right)
$$

This $\mathcal{I}_{m}$ replaces the $t^{m} / m$ ! found when $\Delta$ is constant. For the "linear switching" case, Eq. (2) and $t<T$, the integral above gives (since $\left.f(t)=t^{2} / 2\right), \mathcal{I}_{m}=t^{m+1} /(m+$ $1)$ !. The modified rule for diagrams corresponding to linear switching is to replace $t^{m} / m$ ! by $t^{m+1} /(m+1)$ ! and also add the factor $1 / T$ from Eq. (2). Thus, instead of Eq. (58), taking all relevant diagrams into account we find

$$
c_{n}^{(1)}(t)=\frac{\Delta}{T} \sum_{s=0}^{\infty} \frac{i^{n-1+2 s} t^{n+2 s}(n-1)}{(n+2 s)(n-1+2 s) s !(n-1+s) !}
$$

for $t \leq T$. Note that the phase of this amplitude is $i^{n-1}$. This remains true if we include terms of higher order in $\Delta$. As discussed following Eq. (45), to connect site 1 with site $n$, the corresponding diagrams must have $n-1+2 s$ lines with $s=1,2, \ldots$. Therefore the phase will be $i^{n-1+2 s}$ and terms with odd (even) $n$ will be real (imaginary).

Our next task is to apply these amplitudes to evaluate the sums defined in Eqs. (27), to obtain approximations to the asymptotic amplitudes:

$S_{o}$ to lowest order in $\Delta$ : Since

$$
\left(1+\alpha^{2}\right) q-2 \alpha^{2}=2(q-1)+\Delta^{2}(q-2) \simeq 2(q-1),
$$

to first order in $\Delta$, therefore from Eq. (27)

$$
S_{o}^{(1)}(T)=\Delta^{2}+4 \Delta \sum_{l=1} l c_{2 l+1}^{(1)}(T) .
$$

Inserting the amplitudes from Eq. (61), we find

$$
\begin{aligned}
S_{o}^{(1)}(T)= & \Delta^{2}+4 \frac{\Delta^{2}}{T} \sum_{l=1}^{\infty}(-)^{l} l^{2} \times \\
& \sum_{s=0}^{\infty}(-)^{s} \frac{T^{2(l+s)+1}}{(2(l+s)+1)(l+s) s !(2 l+s) !} \\
= & \frac{\Delta^{2}}{2 T} \sin 2 T,
\end{aligned}
$$

where the steps to perform the double sum are detailed in the Appendix.
$S_{e}$ to lowest order in $\Delta$ : The derivation is entirely similar to that of $S_{o}$, and leads to

$$
\begin{aligned}
S_{e}^{(1)}= & 2 \Delta \sum_{l=1}^{\infty}(2 l-1) c_{2 l}^{(1)}(T) \\
= & -i \frac{\Delta^{2}}{T} \sum_{l=1}^{\infty}(-)^{l}(2 l-1)^{2} \sum_{s=0}^{\infty}(-)^{s} \times \\
& \frac{T^{2(l+s)}}{(l+s)(2(l+s)-1) s !(2 l+s-1) !} \\
= & i \frac{\Delta^{2}}{T} \sin ^{2} T,
\end{aligned}
$$

where the sum is again detailed in the Appendix. To picture how the functions $S_{o}$ and $S_{e}$ behave, we computed them by numerically solving the TDSE to determine the $\vartheta_{q}, q=1,2, \cdots$, and then computed $S_{o}$ and $S_{e}$ (also numerically) using Eqs. (27). Fig. 7 shows the curves thus obtained for three values of $\Delta$. As expected, for the smallest $\Delta$ the curves are very close to the predictions of Eqs. (64) and (65), but even for $\Delta=0.9$, the approximation is qualitatively quite satisfactory. The remaining difference has to be ascribed to contributions of higher order in $\Delta$. Finally, inserting Eqs. (64) and (65)

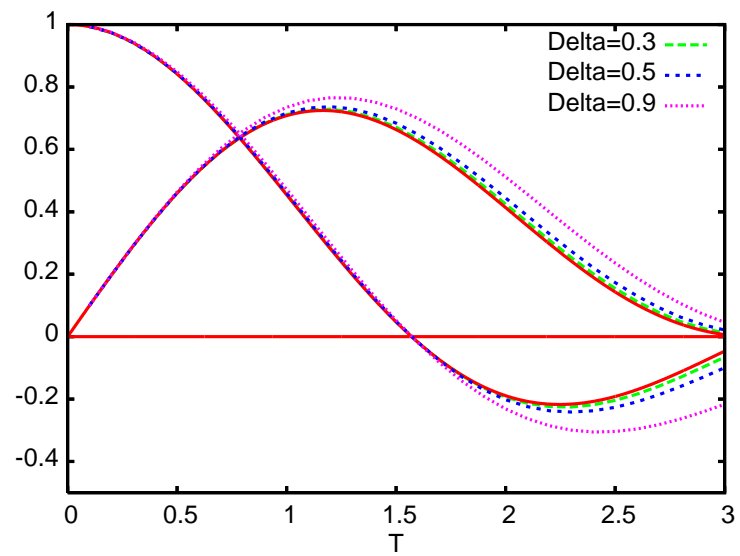

FIG. 7: (Color online) Functions $S_{o}(T) / \Delta^{2}$ and $\operatorname{Im}\left(S_{e}(T) / \Delta^{2}\right)$ for the linear rise model with site 1 initially $(t=0)$ occupied, and the rest empty. Various dashed lines: exact numerical calculations for $\Delta=0.3,0.5$ and 0.9 according to key. Adjacent continuous lines: expressions of Eqs. (64) and (65).

into Eq. (26) we find that to lowest order in $\Delta$ for the $S_{o}(T)$ and $S_{e}(T)$, but keeping $\Delta$ to all orders in the later time-evolution,

$$
\begin{gathered}
c_{1}^{(a)}(t)=\frac{\Delta^{2}}{\sqrt{\pi}(t-T)^{3 / 2}\left(1+\alpha^{2}\right)^{2}} \\
\times \quad \frac{\sin T}{T} \sin \left(2(t-T)-\frac{\pi}{4}+T\right) .
\end{gathered}
$$

The beauty of this expression is that it shows the effect of a finite rise-time $T$, very cleanly: 1 ) it adds a phase 
$T$ to the argument of the sine, and 2) it adds a factor $(\sin T) / T$ to the amplitude of the sudden approximation (the $T \rightarrow 0$ limit.) One sees that for linear switching a finite $T$ always reduces the amplitude. Repeating the same steps for the $c_{n}$, starting from Eq. (20), we arrive at

$$
\begin{aligned}
& c_{n}^{(a)}(t)=\frac{i^{n-1}\left[n+\alpha^{2}(n-2)\right] \Delta}{\sqrt{\pi}(t-T)^{3 / 2}\left(1+\alpha^{2}\right)^{2}} \times \\
& \frac{\sin T}{T} \sin \left(2(t-T)-\left(n-\frac{1}{2}\right) \frac{\pi}{2}+T\right),
\end{aligned}
$$

which shows the same memory effects. Previously, the reduction or enhancement of the amplitude had been discussed only for adiabatic switching: see Robinson, 9] and references therein. Robinson studied the model of a discrete state coupled to a continuum extending from a finite threshold to infinity. The explicit forms of the continuum state density and of the adiabatic switching-on function for discrete to continuum coupling, $f(t)$, were left unspecified. Making only very general assumptions he was able to show that the asymptotic decay was controlled by the Fourier transform of the time-derivative $\dot{f}(t)$, but no results for specific attenuation functions were given. In this paper the continuum is that of the tight binding model; it has finite width, and Robinson's methods do not apply. By working with the Fano-Anderson model, we have obtained explicit forms, Eqs. (66) and (67), for the memory effects.

\section{RESULTS FOR LINEAR SWITCHING}

Amplitudes at $t=T$. To illustrate our results we choose $\Delta=0.3$ as in Fig.1. As has been shown, Eq. (32), the lifetime is $\tau=1 / \gamma=5.3$. We only consider values of the rise time $T \ll \tau$; otherwise the rise and decay regimes would be mixed. We first computed the occupation amplitudes, $c_{n}(T)$, by numerical solution of the time dependent Schrödinger equation as described in 27]. These were compared to the approximate analytical expressions, eq. (61), derived in Section III. The agreement with the exact values of $c_{n}(T)$ is excellent, moreso for small values of $T$. But even at $T=2$ the absolute values are very close: in the format "exact (approximation)", we have for $n=2: 0.222(0.219), n=3: 0.136(0.136)$, $n=4: 0.0676$ (0.0675), $n=5: 0.0275$ (0.0274).

Asymptotic survival probabilities. To compute the asymptotic amplitude $c_{1}^{(a)}(\tilde{t})$. we then use Eqs. (26), (27) and the above exact values for the amplitudes at $t=T$. This is compared to the exact numerical solution of the TDSE in Fig. 8, for the case of rise time $T=1$. Qualitatively similar oscillations are found for other $T$, even as high as $T=2$. In all cases the prediction fom Eq. (28) is excellent. Note also that there is a clear difference from the prediction given by the "sudden approximation" (rise time $T=0$.) We stress that the envelope of the oscillations in $\left|c_{1}^{(a)}(t)\right|^{2}$ follows the predicted $t^{-3}$ algebraic law and that the period of the oscillations, according to Eq. (26) or (28), is $\pi$. Neither result is affected by the finite rise time $T$; only the envelope and phase of the oscillations are affected by the initial-switching process. Finally, we have also plotted the prediction from the approximate expression, eq. 66. On the scale of the figure it cannot be distinguished from the green line previously obtained, thus confirming the accuracy of our approximate analytic expressions. Similarly, we have tested the

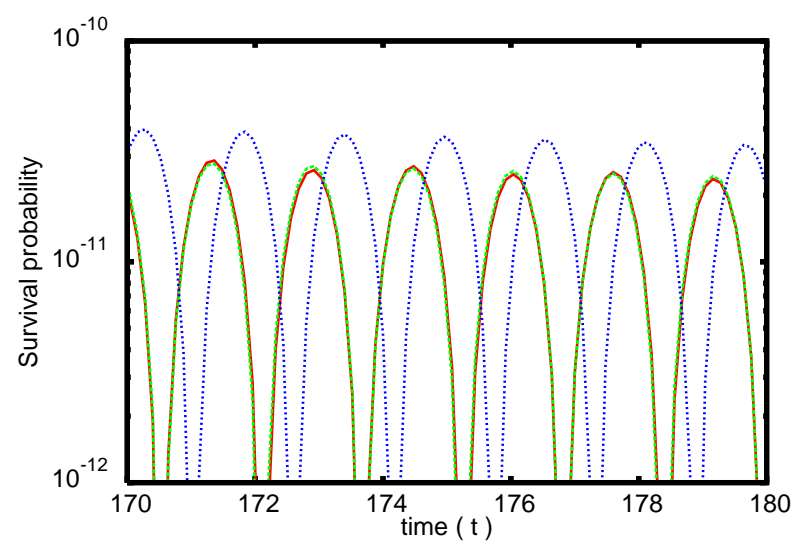

FIG. 8: (Color online) Survival probability in the Linear rise model, for $T=1$ and $\Delta=0.3$. The continuous (red) line is the exact result. Practically hidden by it, the (green) dashed line is the value from the asymptotic expressions in the text. For comparison, the sudden approximation is also shown, as a (blue) dotted line.

accuracy of Eq. (67) by comparison to exact TDSE calculations: The agreement found for the other $c_{n}^{(a)}(t)$ is similar to that for the amplitudes of the first site. Further, we have found that the additional phase $T$ in the argument of the sine, in Eq. (66) has the same value whatever the shape of the switching function, providing that the average value of $\Delta_{v}(t)$ is half of the ultimate value $\Delta$, over the interval $0<t<T$. Finally, we have explored the accuracy of the ansatz $c_{n}(t)=c_{n}^{(e)}(t)+c_{n}^{(a)}(t)$ for $n=1,2, \ldots$ : For $n=1$ and the range of times of Fig. 1, the exact (numerical) result and the values from the ansatz are indistinguishable on the scale of that figure. Similar agreement is found for other small values of $n$. Support for the validity of this ansatz, for the sudden approximation, can be found in the approach based on Laplace transforms presented in [30]: the asymptotic term is an approximation to the contribution due to the Bromwich paths, and to this should be added that of the poles, which produce the exponential decay.

Exponential decay regime In Fig. 9] we compare the exact survival probability $\left|c_{1}\right|^{2}$, again determined from a numerical solution of the TDSE, to the predictions from Eq. (40). Three representative values of $T$ are shown. Except at small times $t \leq T$, the exponential contribution dominates and accurately follows the behaviour of the survival probability. Even for values of $T$ well beyond those suggested by the "sudden approximation" 
condition $(T \Delta \ll 1)[29]$ one sees that the decay is exponential with the same decay constant.

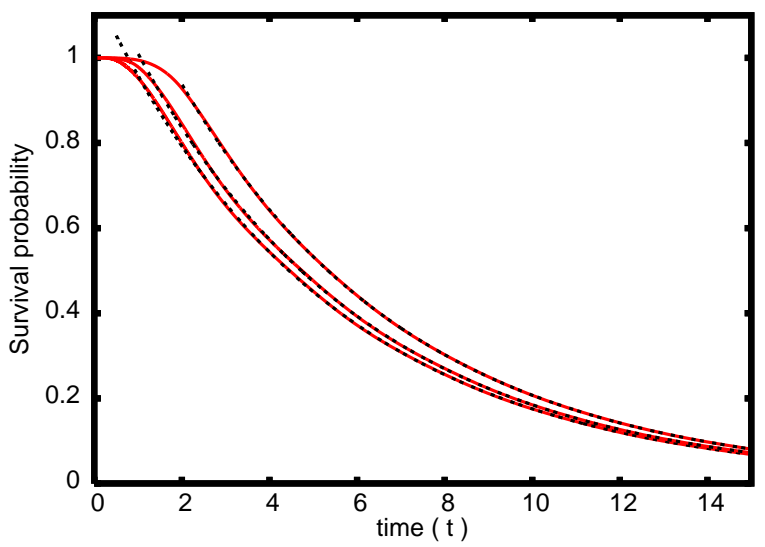

FIG. 9: (Color online) Linear rise model, $\Delta=0.3$ : Survival probabilities for $T=0.5,1$ and 2 from left to right. Continuous (red) lines: exact. Dotted (blue) lines: the exponential decay component, Eq. (40).

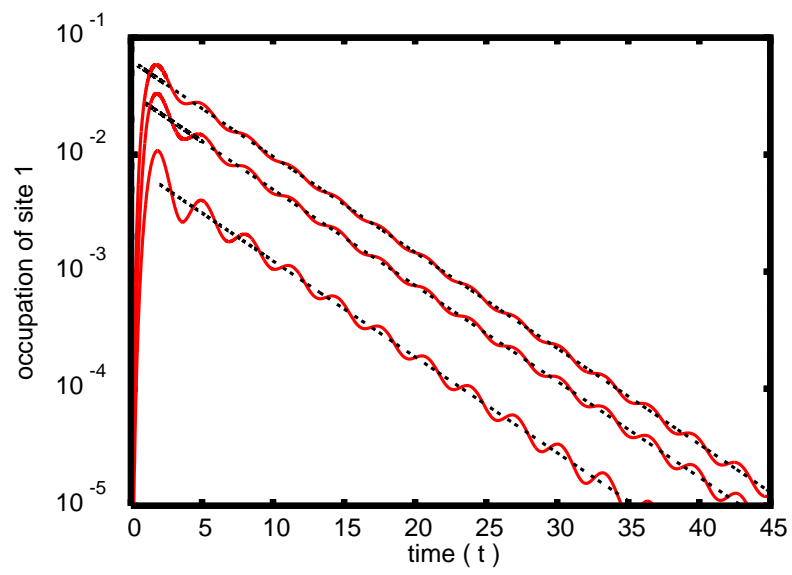

FIG. 10: (Color online) $\left|c_{1}(t)\right|^{2}$ for, from top to bottom, rise times $T=0.5,1$ and 2 . Linear rise model with $\Delta=0.3$, but now site 2 is initially occupied, and the others empty. Continuous (red) lines: exact. Dotted (blue) straight lines: the exponential decay component, Eq. (40).

In Figs. 8 and 9 we assumed that at $t=0$ only the first site was occupied. But, as already stressed, Eqs. (40) are valid for any set of occupation amplitudes at $\tilde{t}=0$. As a second example, we explore what occurs when only site 2 is occupied at $t=0$. Fig. 10] shows the occupation probability $\left|c_{1}\right|^{2}$ for the linear rise model and several values of $T$. Exact (numerical) solutions of the TDSE are compared to values from Eq. (40). (The relevant set of $\vartheta_{q}, q=1,2,$. . was generated by solving the TDSE using $\Delta_{\ell}(t)$.) The exponentially decaying contribution is dominant, except at very small times during which the amplitude $c_{1}(t)$ builds up. The maximum occupation is of order $\Delta^{2}$, and is lower for larger $T$, because site 2 is decaying outwards during the switching-on process before site 1 can begin to fill. Superimposed on the exponential there are pronounced oscillations, due to terms neglected when only the pole at $z_{g}=-i \alpha$ was retained. The average trend is well explained by the exponential.

We have made similar comparisons when initially only site $n=5,10$ or 20 is occupied. In all cases the trend is similar: there is an initial period where the occupation of site 1 accumulates and its subsequent exponential decay is well described by Eq. (40). Increasing the value of $\Delta$ increases the decay constant, so the changeover from exponential to the asymptotic decay regime occurs sooner.

\section{SUMMARY AND DISCUSSION}

In the context of Longhi's version of the FanoAnderson model we have obtained explicit expressions for so-called "memory" effects. In other models these can be studied only numerically or discussed in very general terms, leading to few detailed predictions. The switching-on process occurs during a time interval $T$ between the original preparation of the system in site 1 , and the stabilization of the coupling to the external sites at its final value $g \Delta$. We have shown that no matter what profile $\Delta_{v}(t)$ is chosen, the site amplitudes oscillate asymptotically with period $\pi$, with an envelope having its leading term proportional to $t^{-3 / 2}$. Furthermore, we have also shown that when exponential decay occurs, the decay constant is the same for all site amplitudes.

For the special case of linear switching, we have derived series expansions for the contributions linear in $\Delta$ to the site amplitudes at time $T$, Eq. (61). We have found that to this order the asymptotic amplitude of site 1 is given by Eq. (66), which shows in a very clean way that memory effects introduce a delay $T$ in the phase, and that the modulation of the amplitude is given by a factor $(\sin T) / T$. Combined with the exponentially decaying contribution they allow accurate predictions in the intermediate range of times where interference between the two contributions occurs, and this opens the way to examine memory effects in this range of times.

The methods of Section III, could be applied to quadratic or other forms of initial switching, allowing a systematic study of memory effects. Our predictions for the occupation amplitudes in the initial stages should be amenable to experimental verification using the waveguide analogue of the tight-binding model.

\section{ACKNOWLEDGEMENTS}

We are grateful to DGES-Spain for support through grant FIS2006-10268-C03-01; to UPV-EHU for grant (GIU07/40); and to NSERC-Canada for Discovery grants RGPIN-3198 (DWLS), SAPIN-8672 (WvD). 


\section{APPENDIX A}

To sum the double series in Eq. (64) we make a change of index to $k=l+s$

$$
\begin{aligned}
D & \equiv \sum_{l=1}(-)^{l} l^{2} \sum_{s=0}(-)^{s} \frac{T^{2(l+s)+1}}{(2(l+s)+1)(l+s) s !(2 l+s) !} \\
& =\sum_{k=1}^{\infty}(-)^{k} \frac{T^{2 k+1}}{k(2 k+1)} \sum_{s=0}^{k} \frac{(k-s)^{2}}{s !(2 k-s) !}
\end{aligned}
$$

and to perform the sum over $s$, we write

$$
\begin{aligned}
\sum_{s=0}^{k} \frac{(k-s)^{2}}{s !(2 k-s) !} & =\sum_{s=0}^{2 k} \frac{(k-s)^{2}}{s !(2 k-s) !}-\sum_{s=k+1}^{2 k} \frac{(k-s)^{2}}{s !(2 k-s) !} \\
& =\sum_{s=0}^{2 k} \frac{(k-s)^{2}}{s !(2 k-s) !}-\sum_{s^{\prime}=0}^{k-1} \frac{\left(k-2 k+s^{\prime}\right)^{2}}{\left(2 k-s^{\prime}\right) !\left(s^{\prime}\right) !} \\
& =\sum_{s=0}^{2 k} \frac{(k-s)^{2}}{s !(2 k-s) !}-\sum_{s=0}^{k-1} \frac{(k-s)^{2}}{s !(2 k-s) !}(\mathrm{A} 2)
\end{aligned}
$$

where we have introduced $s^{\prime}=2 k-s$ to rewrite the second sum. Next, noticing that

$$
\sum_{s=0}^{k} \frac{(k-s)^{2}}{s !(2 k-s) !}=\sum_{s=0}^{k-1} \frac{(k-s)^{2}}{s !(2 k-s) !}
$$

we finally arrive at

$$
\sum_{s=0}^{k} \frac{(k-s)^{2}}{s !(2 k-s) !}=\frac{1}{2} \sum_{s=0}^{2 k} \frac{(k-s)^{2}}{s !(2 k-s) !}
$$

Using the identities

$$
\begin{aligned}
\sum_{s=0}^{2 k}\left(\begin{array}{c}
2 k \\
s
\end{array}\right) & =2^{2 k} \\
\sum_{s=0}^{2 k} s\left(\begin{array}{c}
2 k \\
s
\end{array}\right) & =2^{2 k} k \\
\sum_{s=0}^{2 k} s(s-1)\left(\begin{array}{c}
2 k \\
s
\end{array}\right) & =2^{2 k-1} k(2 k-1)
\end{aligned}
$$

and decomposing the numerator of Eq. (A3) as $(k-s)^{2}=$ $k^{2}-(2 k-1) s+s(s-1)$, Eq. (64) simplifies to

$$
S_{o}^{(1)}(T)=\frac{\Delta^{2}}{2 T} \sum_{k=0}(-)^{k} \frac{(2 T)^{2 k+1}}{(2 k+1) !}=\Delta^{2} \frac{\sin 2 T}{2 T} \text {. }
$$

The double series in Eq. [65) is summed using the same methods: We again write $k=l+s$ to replace the sums over $l$ and $s$ by sums over $k$ and $s$ :

$$
S_{e}^{(1)}=-i \frac{\Delta^{2}}{T} \sum_{k=1}^{\infty} \frac{(-)^{k} T^{2 k}}{k(2 k-1)} \sum_{s=0}^{k-1} \frac{(2 k-2 s-1)^{2}}{s !(2 k-s-1) !}
$$

and to evaluate the sum over $s$ we proceed as before

$$
\begin{aligned}
& \sum_{s=0}^{k-1} \frac{(2 k-1-2 s)^{2}}{s !(2 k-1-s) !} \\
= & \sum_{s=0}^{2 k-1} \frac{(2 k-1-2 s)^{2}}{s !(2 k-1-s) !}-\sum_{s=k}^{2 k-1} \frac{(2 k-1-2 s)^{2}}{s !(2 k-1-s) !} \\
= & \sum_{s=0}^{2 k-1} \frac{(2 k-1-2 s)^{2}}{s !(2 k-1-s) !}-\sum_{s^{\prime}=0}^{k-1} \frac{\left(2 k-1-2\left(2 k-1-s^{\prime}\right)\right)^{2}}{\left(s^{\prime}\right) !\left(2 k-1-s^{\prime}\right) !} \\
= & \sum_{s=0}^{2 k-1} \frac{(2 k-1-2 s)^{2}}{s !(2 k-1-s) !}-\sum_{s=0}^{k-1} \frac{(2 k-1-2 s)^{2}}{s !(2 k-1-s) !}
\end{aligned}
$$

and therefore

$$
\sum_{s=0}^{k-1} \frac{(2 k-1-2 s)^{2}}{s !(2 k-1-s) !}=\frac{1}{2} \sum_{s=0}^{2 k-1} \frac{(2 k-1-2 s)^{2}}{s !(2 k-1-s) !}
$$

Next we decompose the numerator as

$$
(2 k-1-2 s)^{2}=(2 k-1)^{2}-8(k-1) s+4 s(s-1)
$$

and use Eq. (A5), with replacement $2 k \rightarrow 2 k-1$ to rewrite the sum as

$$
\sum_{s=0}^{k-1} \frac{(2 k-1-2 s)^{2}}{s !(2 k-1-s) !}=\frac{2^{2 k-2}}{(2 k-2) !}
$$

We now insert this in Eq. (A7) and find

$$
\begin{aligned}
S_{e}^{(1)} & =i \frac{\Delta^{2}}{2 T} \sum_{k=1}^{\infty}(-)^{k} \frac{(2 T)^{2 k}}{(2 k) !} \\
& =i \frac{\Delta^{2}}{2 T}(1-\cos 2 T)=i \frac{\Delta^{2}}{T} \sin ^{2} T .
\end{aligned}
$$

[1] M. Razavy, "Quantum Theory of Tunnelling", ISBN 981238-018-3, (World Scientific, 2003). The first chapter has a succint summary of the early history of quantum tunneling.
[2] L. A. Khalfin, Zh. Eksper. Theor. Fiz. 33, 1371-82 (1957); Sov. Phys. JETP 6, 1053-63 (1958).

[3] R. G. Winter, Phys. Rev. 123, 1503-7 (1961).

[4] M. L. Goldberger and K.M. Watson, "Collision Theory", 
(John Wiley and Sons, NY, 1964). See pp. $270 \mathrm{ff}$.

[5] J. Martorell, J.G. Muga and D.W.L. Sprung, Phys. Rev. A 77, 042719 (2008).

[6] J. Martorell, J.G. Muga and D.W.L. Sprung, "Quantal Post-exponential Decay" in "Time in Quantum Mechanics", vol. II; (Springer, to be published).

[7] C. Cohen-Tannoudji, J. Dupont-Roc and G. Grynberg, "Atom-Photon Interactions: Basic Processes and Applications", (Wiley, NY, 1992).

[8] H. Nakazato, M. Namiki and S. Pascazio, Int. J. Mod. Phys. B 10, 247-295 (1996); P. Facchi and S. Pascazio, "La Regola d'oro di Fermi", (ISBN 88-7088-304-3, Bibliopolis, 1999).

[9] E.J. Robinson, Phys. Rev. A 33, 1461-3 (1986).

[10] N.W. Ashcroft and N.D. Mermin, "Solid State Physics", Holt Saunders International Editions, 1976.

[11] C. Kittel, "Introduction to Solid State Physics", 7th edition, 1996. John Wiley and Sons.

[12] J.H. Davies, "The Physics of Low Dimensional Semiconductors", Cambridge U.P. (1999).

[13] T. Hartmann, F. Keck, H.J. Korsch and S. Mossmann, New Journal of Physics 6(2004)2.

[14] A. Smerzi and A. Trombettoni, Phys. Rev. A68(2003)023613.

[15] U. Fano, Phys. Rev. 124, 1866-78 (1961).

[16] P.W. Anderson, Phys. Rev. 124, 41-53 (1961).
[17] G.D. Mahan, "Many-Particle Physics", Second edn., (Plenum Press, New York, 1990), pp. 272-285.

[18] S. Longhi, Phys. Rev. Lett. 97, 110402 (2006).

[19] P. Facchi and S. Pascazio, J. Phys. A 41, 493001 (2008).

[20] S. Longhi, Phys. Rev. E 74, 026602 (2006).

[21] A.L. Jones, J. Opt. Soc. Am. 55, 261-271 (1965).

[22] D.N. Christodoulides, F. Lederer and Y. Silverberg, Nature 424, 817-23 (2003).

[23] G. Della Valle, S. Longhi, P. Laporta, P. Biagioni, L. Dou and M. Finazzi, Appl. Phys. Lett. 90, 261118 (2007).

[24] P. Biagoni, G. Della Valle, M. Ornigotti, M. Finazzi, L. Duo, P. Laporta and S. Longhi, Optics Express 16, 37627 (2008).

[25] A. Raczynski and J. Zaremba, Phys. Reports 235, 1-55 (1993).

[26] S. Longhi, G. Della Valle, M.Ornigotti and P. Laporta, Phys. Rev. B 76, 201101(R) (2007)

[27] S.E. Koonin and D.C. Meredith, "Computational Physics", (Addison-Wesley, 1990), pp.186-188.

[28] G. Arfken, "Mathematical Methods for Physicists", Third Edn., (Academic Press, 1985).

[29] A. Messiah, "Quantum Mechanics", (Dover reprint 1999), pp. 739-742.

[30] S. Longhi, Phys. Rev. A 74, 063826 (2006).

file: initex.tex May 11, 2009 